\title{
Female pelvic floor dysfunction questionnaires: the modern Tower of Babel?
}

\author{
Themos Grigoriadis $^{1} \cdot$ Stavros Athanasiou ${ }^{1} \cdot$ Diaa Rizk $^{2}$
}

Accepted: 24 December 2019 / Published online: 27 January 2020

(C) The International Urogynecological Association 2020

Keywords Pelvic floor dysfunctions $\cdot$ Patient reported outcomes $\cdot$ Questionnaires $\cdot$ Tower of Babel

Female pelvic floor dysfunctions (PFDs) are a wide spectrum of prevalent conditions such as pelvic organ prolapse (POP), urinary incontinence, voiding and/or defecation dysfunction, sexual dysfunction and chronic pelvic pain syndromes [1]. Evaluation of women presenting with PFD includes careful analysis of symptoms, their impact on quality of life and daily activities and targeted physical examination. It is generally accepted, however, that clinical assessment is inherently subjective and is, therefore, prone to bias.

According to the International Consultation on Incontinence (ICI), the most valid measure of the presence, severity and impact of PFD on patient's activities and wellbeing is psychometrically validated and self-completed patient questionnaires or patient-reported outcome measures (PROMs) [2]. These are defined by The Food and Drug Administration (FDA) as "any report of the status of health condition that comes directly from the patient, without interpretation of the patient's response by a clinician or anyone else" [3], representing the most important clinical appraisal of symptom impact and treatment benefit from the patient's perspective. PROM offers, therefore, an objective assessment of PFD allowing standardized collection of data for audit and research to compare between different interventions.

Over the last 3 decades, many both generic and conditionspecific PROM questionnaires have been developed for use in daily practice as well as clinical research. This multitude of published PROMs, however, has presented a challenging task

Themos Grigoriadis tgregos@yahoo.com

1 Urogynecology Unit, First Department of Obstetrics and Gynecology, "Alexandra Hospital”, National and Kapodistrian University of Athens, 80 Vas. Sofias Avenue, 11528 Athens, Greece

2 Department of Obstetrics and Gynecology, College of Medicine and Medical Sciences, Arabian Gulf University, Manama, Bahrain for urogynecologists trying to become familiar with the content, layout and scoring algorithms of each questionnaire to choose the most appropriate instrument. In fact, there are five dedicated chapters covering PROM in the Textbook of Female Urology and Urogynecology, one of the comprehensive texts on urogynecology. In these chapters, 105 PFD questionnaires are described concerning health-related quality of life (HRQL), symptom bother, urgency-specific measures, screener satisfaction, goal assessment tools, bowel dysfunction, sexual dysfunction, POP and the Electronic Personal Assessment Questionnaire Pelvic Floor [4]. Similarly, in 2017, the 6th ICI recommended using 33 HRQL measures for lower urinary tract symptoms (LUTS), 8 patient satisfaction questionnaires, 17 screening tools and 12 symptom bother measures for LUTS, 13 urinary urgency measures, 10 measures for fecal incontinence, other bowel symptoms and HRQL-associated and 5 sexual health and quality of life measures [5]. One can easily understand the difficulty for and perplexity of clinicians trying to access, retrieve and review each questionnaire and then decide on the one to use for a specific pelvic floor disorder.

The diversity of available PFD questionnaires can also limit the ability to compare clinical and research data, thus defeating the original purpose of developing PROM. For example, the ten most recently published randomized trials in the International Urogynecology Journal reporting the use of PROM to evaluate different treatments of POP include $>25$ PROM questionnaires. In these studies, prolapse symptoms alone were assessed by seven different PROMs and LUTS by six [6-15]. The resulting confusion and difficulty when comparing outcomes are obvious, as each questionnaire examines specific psychometric properties and is scored by different numerical values. Interestingly, many of the abovementioned trials evaluate the efficacy of interventions on responses of single questions of the questionnaires rather than by comparing scores of PROMs [6-11]. Furthermore, a 
statistically significant difference in scores may not necessarily correlate to a clinically meaningful response or a subjectively important outcome. Hence, it is imperative to know the smallest change in PROM scores for each questionnaire that patients consider important, defined as the minimal important difference (MID) [16]. It is rare to find studies with reported MIDs for each PROM questionnaire in the urogynecologic literature as only a few were designed to identify this value.

Most PROM questionnaires were developed and validated in English-speaking populations. This limits the reliability of using the questionnaires in different populations, clinical settings and languages. Validated translation and cross-cultural adaptation of PROM are thus required to ensure psychometric adaptation and provide conceptual, idiomatic and content equivalence with the original questionnaire. The International Urogynecological Association/International Continence Society joint report on the terminology for reporting outcomes of surgical procedures for pelvic organ prolapse highlights the importance of this point and recommends validation of study questionnaires in the language of the trial investigator(s) [17]. Only a few of the available PROMs, however, have been translated and cross-culturally validated, restricting their use among different patient populations. It is noteworthy that the Pelvic Organ Prolapse Symptom Score (POP-SS) questionnaire used in the PROSPECT trial has been translated in only two other languages [18].

In conclusion, the large number of PROM questionnaires that are currently used to assess female PFD could be paralleled to the narrative of the Tower of Babel (Fig. 1). In the biblical literature, humanity speaking a single language started to build a tower tall enough to reach heaven. However, as people suddenly spoke different languages, they lost the ability to understand each other and could not communicate and work together. As a result, the tower was never completed. Likewise, the PROM questionnaires used in

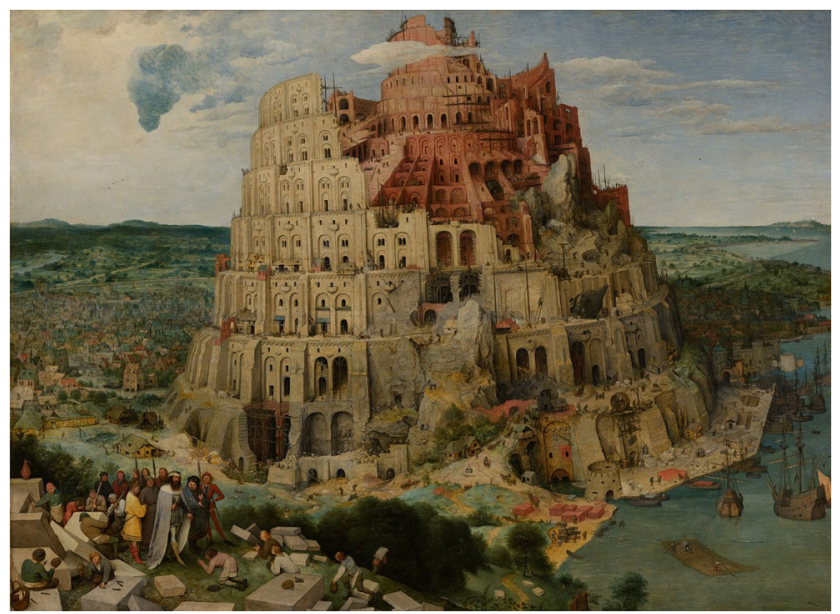

Fig. 1 The Tower of Babel, Pieter Bruegel the Elder, Kunsthistorisches Museum, Vienna, Austria research and clinical practice nowadays, with the lack of validated translation and cultural adaptation of the majority, jeopardize the objective and standardized assessment of PFD across different populations. This may annul one of the principal objectives of constructing and using PROM with the risk of having "a modern Babel tower of questionnaires." We believe that international bodies and professional societies involved in urogynecology should develop an updated and evidence-based consensus and/or guidelines on the value of only a selected number of PROM questionnaires to resolve the problem. In this way, we will continue to speak and understand the same language.

Author contribution to the manuscript T. Grigoriadis: Conception of the topic, Manuscript writing.

Stavros Athanasiou: Manuscript Editing.

Diaa Rizk: Manuscript Editing.

\section{Compliance with ethical standards}

Conflict of interest None.

\section{References}

1. Bump RC, Norton PA. Epidemiology and natural history of pelvic floor dysfunction. Obstet Gynecol Clin N Am. 1998;25:723-46.

2. Maher C, Baessler K, Barber M, Cheon C, Consten E, et al. Committee 15. Pelvic organ prolapse surgery. In: Abrams P, Cardozo L, Wagg A, Wein A, editors. Incontinence. 6th ed. Bristol UK: ICI-ICS International Continence Society; 2017. p. 1858-992.

3. Food and Drug Administration (FDA): Guidance for Industry on Patient-Reported Outcome Measures: Use in Medical Product Development to Support Labeling Claims. Guidance for Industry. Clinical - Medical, Good Clinical Practices. 2009. https://www.fda. gov/media/77832/download. Accessed 20 July 2019

4. Cardozo L, Staskin D. Textbook of female urology and Urogynecology. Boca Raton: CRC Press; 2017.

5. Abrams P, Cardozo L, Wagg A, Wein A. Incontinence 6th edition. ICI-ICS. International Continence Society, Bristol UK; 2017.

6. Steures P, Milani A, Kluivers K, Withagen M. Partially absorbable mesh or native tissue repair for pelvic organ prolapse: a randomized controlled trial. Int Urogynecol J. 2019;30(4):565-73.

7. Bataller $\mathrm{E}$, Ros $\mathrm{C}$, Anglès $\mathrm{S}$, Gallego $\mathrm{M}$, Espuña-Pons $\mathrm{M}$, Carmona F. Anatomical outcomes 1 year after pelvic organ prolapse surgery in patients with and without a uterus at a high risk of recurrence: a randomised controlled trial comparing laparoscopic sacrocolpopexy/cervicopexy and anterior vaginal mesh. Int Urogynecol J. 2019;30(4):545-55.

8. Milani A, Damoiseaux A, IntHout J, Kluivers K, Withagen M. Long-term outcome of vaginal mesh or native tissue in recurrent prolapse: a randomized controlled trial. Int Urogynecol J. 2018;29(6):847-58.

9. Chattot C, Deffieux X, Lucot J, Fritel X, Fauconnier A. Preoperative predictors and a prediction score for perception of improvement after mesh prolapse surgery. Int Urogynecol J. 2019;1-8.

10. Due U, Brostrøm S, Lose G. Lifestyle advice with or without pelvic floor muscle training for pelvic organ prolapse: a randomized controlled trial. Int Urogynecol J. 2016;27(4):555-63. 
11. Ow L, Lim Y, Lee J, Murray C, Thomas E, Leitch A, et al. RCT of vaginal extraperitoneal uterosacral ligament suspension (VEULS) with anterior mesh versus sacrocolpopexy: 4-year outcome. Int Urogynecol J. 2018;29(11):1607-14.

12. Rondini C, Braun H, Alvarez J, Urzúa M, Villegas R, Wenzel C, et al. High uterosacral vault suspension vs Sacrocolpopexy for treating apical defects: a randomized controlled trial with twelve months follow-up. Int Urogynecol J. 2015;26(8):1131-8.

13. Rahmanou P, Price N, Jackson S. Laparoscopic hysteropexy versus vaginal hysterectomy for the treatment of uterovaginal prolapse: a prospective randomized pilot study. Int Urogynecol J. 2015;26(11): 1687-94.

14. Geynisman-Tan J, Kenton K, Komar A, Collins S, Lewicky-Gaupp C, Mueller M. Recovering sexual satisfaction after prolapse surgery: a secondary analysis of surgical recovery. Int Urogynecol J. 2018;29(11):1675-80.

15. de Castro E, Brito L, Juliato C (2019). Vaginal hysterectomy with bilateral sacrospinous fixation plus an anterior mesh versus abdominal sacrocervicopexy for the treatment of primary apical prolapse in postmenopausal women: a randomized controlled study. Int Urogynecol J. https://doi.org/10.1007/s00192-019-03948-3.

16. Coyne K, Sexton C. Patient reported outcomes: from development to utilization. In: Cardozo L, Staskin D, editors. Textbook of female urology and Urogynecology. Boca Raton: CRC Press; 2017. p. 8895 .

17. Toozs-Hobson P, Freeman R, Barber M, Maher C, Haylen B, et al An international Urogynecological association (IUGA)/international continence society (ICS) joint report on the terminology for reporting outcomes of surgical procedures for pelvic organ prolapse. Int Urogynecol J. 2012;23:527-35.

18. Glazener CM, Breeman S, Elders A, Hemming C, Cooper KG, et al. Mesh, graft, or standard repair for women having primary transvaginal anterior or posterior compartment prolapse surgery: two parallel-group, multicentre, randomised, controlled trials (PROSPECT). Lancet. 2017;389:381-92.

Publisher's note Springer Nature remains neutral with regard to jurisdictional claims in published maps and institutional affiliations. 